О. Г. Гоман, В. И. Карплюк

Днепровский национальный университет имени Олеся Гончара

\title{
РАСЧЕТ ПАРАМЕТРОВ ТЕЧЕНИЯ В ДИФФУЗОРЕ С УЧЕТОМ ВЗАИМНОГО ВЛИЯНИЯ ПОГРАНИЧНОГО СЛОЯ И ЯДРА ПОТОКА
}

Диффузор представляет собой устройство, предназначенное для преобразования кинетической энергии потока в потенциальную энергию за счет геоетрического воздействия на поток. Преобразование энергии в диффузоре сопровождается возрастанием энтропии и уменьшением полного давления.

В данной работе представлен приближенный метод расчета течения в диффузоре с учетом наличия пограничного слоя на стенках и ядра потока, а также их взаимного влияния друг на друга. Использованная модель основана на рассмотрении интегральных законов сохранения массы, импульса и энергии и учитывает эффекты взаимного влияния ядра течения и пограничного слоя. Для расчета параметров пограничного слоя используются интегральные методы типа Кармана. Течение в диффузоре предполагается адиабатичным но неизэнтропичным. Полная энергия при этом не меняется, но за счет сил трения происходит перераспределение между кинетической и тепловой энергией. Учитывая, что в поперечном сечении поток состоит из двух областей: ядра и кольцевого пограничного слоя, из законов сохранения массы, импульса и энергии получена система из четырех уравнений для определения параметров потока в ядре диффузора, из которых лишь одно является дифференциальным, а остальные - конечные.

Предложенная методика расчета основана на использовании общих интегральных уравнений закона сохранения массы, импульса и энергии для канала с учетом наличия пограничного слоя на стенках и ядра потока и их взаимного влияния друг на друга.

Приведенные результаты расчетов позволяют выбрать параметры диффузора, обеспечивающие необходимый расход и рассчитанного на определенную величину давления в выходном сечении.

Ключевые слова: диффузор, приближенная методика расчета, законы сохранения, пограничный слой.

Дифузор є пристроєм, призначеним для перетворення кінетичної енергії потоку в потенційну енергію за рахунок геометричної дії на потік. Перетворення енергії в дифузорі супроводжується зростанням ентропії і зменшенням повного тиску.

У цій роботі представлений наближений метод розрахунку течії в дифузорі з урахуванням наявності пограничного шару на стінках і ядра потоку, а також їх взасмного впливу один на одного. Використана модель грунтусться на розгляді інтегральних законів збереження маси, імпульсу і енергії і враховує ефекти взаємного впливу ядра течії і пограничного шару. Для розрахунку параметрів пограничного шару використовуються інтегральні методи типу Кармана. Течія в дифузорі передбачається адіабатичною, але неізентропічною. Повна енергія при цьому не мінясться, але за рахунок сил тертя відбувається перерозподіл між кінетичною і тепловою енергією. Враховуючи, що в поперечному перерізі потік складається 3 двох областей: ядра і кільцевого пограничного шару, із законів збереження маси, імпульсу і енергії отримана система з чотирьох рівнянь для визначення параметрів потоку в ядрі дифузора, 3 яких лише одно є диференціальним, а інші - кінцеві.

Запропонована методика розрахунку грунтусться на використанні загальних інтегральних рівнянь закону збереження маси, імпульсу і енергії для каналу з урахуванням наявності пограничного шару на стінках і ядра потоку і їх взаємного впливу один на одного.

Приведені результати розрахунків дозволяють вибрати параметри дифузора, що забезпечують необхідну витрату і розрахованого на певну величину тиску у вихідному перерізі.

Ключові слова: дифузор, наближена методика розрахунку, закони збереження, пограничний шар. 
A diffuser is the device intended for transformation of kinetic energy of stream to potential energy due to the geometrical affecting stream. Transformation of energy to the diffuser is accompanied by growth of entropy and reduction of complete pressure.

In hired the close method of calculation of flow is presented in a diffuser taking into account the presence of frontier layer on walls and kernel of stream, and also their cross-coupling on each other.

The used model is based on consideration of integral laws of maintenance of mass, impulse and energy and takes into account the effects of cross-coupling of kernel of flow and frontier layer. For the calculation of parameters of frontier layer the integral methods of type of Pocket are used. A flow in a diffuser is assumed as adiabatic but not isentropic. Complete energy does not change here, but due to forces of friction there is a redistribution between kinetic and thermal energy. Taking into account that in a crossrunner a stream consists of two areas: kernel and circular frontier layer, from the law of maintenance of mass, impulse and energy the system is got from four equalizations for determination of parameters of stream in the kernel of diffuser, from that only one is differential, and other - eventual.

An offer methodology of calculation is based on the use of general integral equalizations of law of maintenance of mass, impulse and energy for a channel taking into account the presence of frontier layer on walls and kernel of stream and their cross-coupling on each other.

The brought results over of calculations allow to choose the parameters of diffuser, providing a necessary expense and the pressure counted on a certain size in an output section.

Key words: diffuser, approximate calculation technique, conservation laws, boundary layer.

Введение. При изучении течения газа в трубах и каналах (в частности, диффузорах) различают два различных участка течения: начальный (входной) и основной участки. Начальный участок характеризуется непрерывной деформацией профиля скорости по потоку. Вначале масса втекающего газа не испытывает существенного влияния вязкости. На стенках канала, начиная от входного сечения, образуется пограничный слой, толщина которого постепенно нарастает, а потенциальное ядро потока сужается. Расстояние от входного сечения до того сечения, в котором пограничные слои со всех сторон смыкаются (а ядро исчезает) называется длиной начального участка. В работе рассматриваются короткие диффузоры, длина которых меньше длины начального участка. В коротком диффузоре пограничный слой достаточно тонок по сравнению с диаметром диффузора. Поэтому, для коротких диффузоров можно пользоваться теорией пограничного слоя и пренебрегать пространственными эффектами, полагая пограничный слой плоским.

В данной работе представлен приближенный метод расчета течения в диффузоре с учетом наличия пограничного слоя на стенках и ядра потока, а также их взаимного влияния друг на друга.

Цель работы. Разработка приближенной методики расчета, позволяющей оперативно производить расчеты потерь давления в диффузорах во всем диапазоне чисел Маха.

Все известные инженерные подходы расчета диффузоров [1-6] являются одномерными и, строго говоря применимы только к прямолинейным диффузорам с круговым поперечным сечением. Расчет криволинейного диффузора с некруговым поперечным сечением сводится к расчету эквивалентного «распрямленного» диффузора с круговым поперечным сечением.

Схема осесимметричного диффузора с прямолинейной осью представлена на рис.1. 


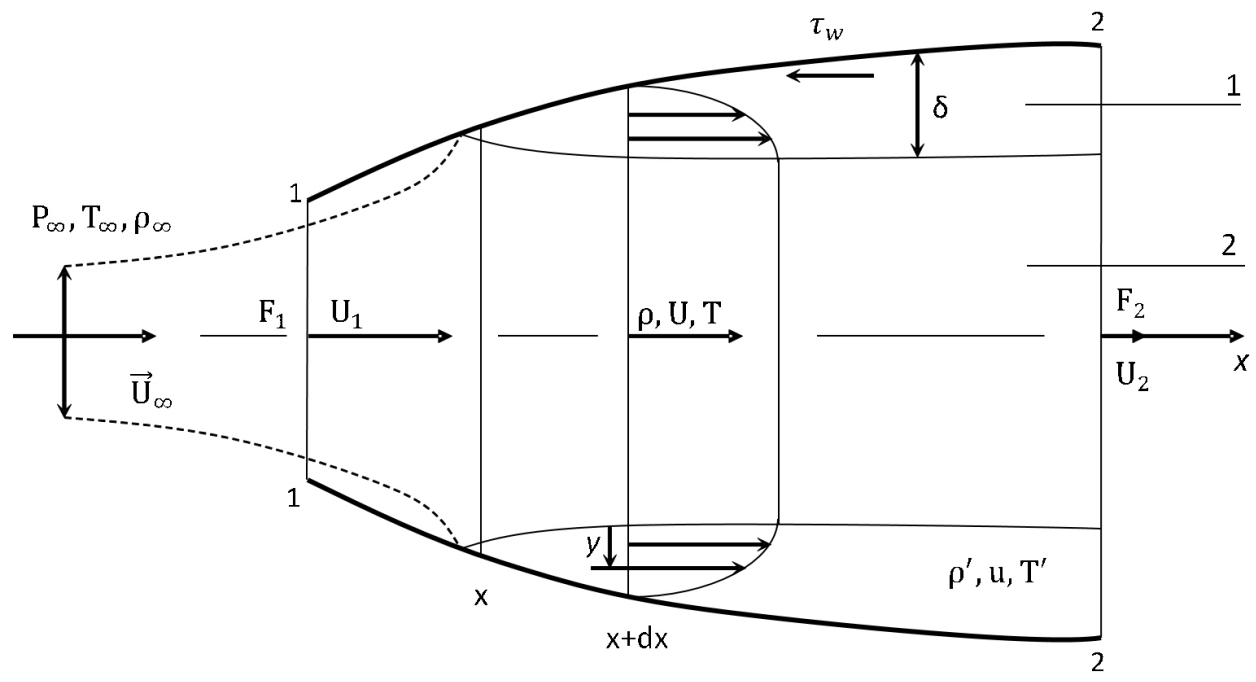

Рис. 1. Схема течения в диффузоре:

1 - пограничный слой; 2 - ядро потока

Скорость в ядре потока обозначим через $\mathrm{U}$, плотность $\rho$, давление $P$. Внутри пограничного слоя - скорость $u$, плотность $\rho^{\prime}$. Площадь поперечного сечения диффузора $F(x)=\pi D^{2} / 4$.

Рассмотрим участок потока в диффузоре между двумя поперечными сечениями $x$ и $x+d x$. Применим к этому участку законы сохранения массы, импульса и энергии:

$$
\begin{gathered}
\int_{F} \rho u d F=\dot{m} \\
\frac{\partial}{\partial x} \int_{F} \rho u^{2} d F+F \frac{\partial P}{\partial x}=-\tau_{w} \chi \\
\frac{\partial}{\partial x} \int_{F} \rho u\left(i+\frac{1}{2} u^{2}\right) d F=0
\end{gathered}
$$

где $\dot{m}$ - заданный (потребный) секундный расход массы через диффузор; $\chi \chi-$ периметр в рассматриваемом сечении; $\tau_{w}$ - напряжение трения на стенке.

При выводе уравнения энергии (3) предполагалось, что стенки диффузора теплоизолированы, поэтому работа сил трения превращается в тепло, которое остается в объеме. Указанные величины (работа сил трения и тепло) взаимно компенсируют друг друга и поэтому в уравнении (3) соответствующие члены не проявляются. Таким образом, течение в диффузоре считается адиабатичным, но неизоэнтропичным. Полная энергия при этом не меняется, но за счет трения происходит перераспределение между кинетической и тепловой энергией. 
Учитывая, что в поперечном сечении поток состоит из двух областей: ядра и кольцевого пограничного слоя, из закона сохранения массы (1) получим

$$
\rho U\left(F-F_{1}^{*}\right)=\dot{m}
$$

где $F_{1}^{*}$ - площадь вытеснения, равная

$$
F_{1}^{*}=\int_{0}^{\delta}\left(1-\frac{\rho^{\prime} u}{\rho U}\right)(R-y) d y
$$

Переменной «у» обозначено расстояние от стенки внутрь пограничного слоя. Предполагая, что пограничный слой тонок $\delta / R<<1$ (короткие диффузоры), величину $(R-y)$ под интегралом (5) можно заменить на $R$, и тогда, учитывая определение толщины вытеснения [2], получим

$$
F_{1}^{*}=2 \pi R \delta_{1}
$$

Величина $F_{1}^{*}$ представляет собой кольцевую площадь вытеснения потока, за счет которой уменьшается проходное сечение в ядре.

Из теорем импульсов получаем уравнение

$$
\frac{\partial}{\partial x} \rho U^{2}\left(F-F_{1}^{*}-F_{2}^{* *}\right)+F \frac{\partial P}{\partial x}=-\tau_{w} \chi,
$$

где $F_{1}^{*}$ - площадь вытеснения (5), а $F_{2}^{* *}$ - площадь потери импульса

$$
F_{2}^{* *}=\int_{0}^{\delta} \frac{\rho^{\prime} u}{\rho U}\left(1-\frac{u}{U}\right)(R-y) d y .
$$

В предположении, что $\delta / R<<1$, имеем

$$
F_{1}^{*}+F_{2}^{* *}=2 \pi R\left(\delta_{1}+\delta_{2}\right)
$$

Наконец, из уравнения энергии получим

$$
\rho U F\left(i+\frac{U^{2}}{2}\right)-\rho U i\left(F_{1}^{*}-F_{H}^{*}\right)-\frac{\rho U^{3}}{2}\left(F_{1}^{*}+F_{2}^{* *}\right)=C,
$$

где $F_{H}^{*}$ площадь увеличения энтропии

$$
F_{H}^{*}=2 \pi \int_{0}^{\delta} \frac{\rho^{\prime} u}{\rho U}\left(\frac{i^{\prime}}{i}-1\right)(R-y) d y \approx 2 \pi R \delta_{H}
$$


и $\delta_{H}$ - толщина увеличения энтальпии.

Рассмотрим ту часть струи, поступающей в диффузор, которая расположена далеко впереди перед диффузором (рис.1). Согласно (4)

$$
\dot{m}=\rho_{\infty} U_{\infty} F_{\infty}=\rho_{1} U_{1} F_{1},
$$

где $F_{\infty}$ - площадь поперечного сечения струи в бесконечности, $\rho_{\infty}$-атмосферная плотность и $U_{\infty}-$ скорость набегающего потока. Правая часть в уравнении (12) имеет место потому, что во входном сечении пограничный слой еще отсутствует. Из (12) имеем

$$
\frac{F_{\infty}}{F_{1}}=\frac{\rho_{1} U_{1}}{\rho_{\infty} U_{\infty}} .
$$

Эксперименты показывают, что для дозвукового диффузора оптимальными режимами являются такие, когда [3]

$$
\frac{U_{1}}{U_{\infty}} \approx 0,5 .
$$

Отсюда, учитывая, что плотность в дозвуковом потоке меняется незначительно, из (13) можно заключить, что наиболее выгодным является такой режим, когда

$$
\frac{F_{\infty}}{F_{1}} \approx 0,5,
$$

то есть струя еще перед входом в диффузор должна расширяться (тормозиться), как показано на рис. 1.

Применяя уравнение энергии (10) к сечению в бесконечности перед диффузором, определим значение константы:

$$
\begin{gathered}
C=\rho_{\infty} U_{\infty} F_{\infty}\left(i_{\infty}+\frac{U_{\infty}^{2}}{2}\right)=\dot{m}\left(i_{\infty}+\frac{U_{\infty}^{2}}{2}\right)=\dot{m}\left(c_{P} T_{\infty}+\frac{U_{\infty}^{2}}{2}\right)= \\
=\dot{m}\left(\frac{k}{k-1} \frac{P_{\infty}}{\rho_{\infty}}+\frac{U_{\infty}^{2}}{2}\right)=\dot{m} i_{0} .
\end{gathered}
$$

Система уравнений (4), (7), (10) совместно с уравнениями для расчета пограничного слоя [4] служит для расчета параметров течения в диффузоре.

Уравнение сохранения масс (4) и энергии (10) представляет собой алгебраические уравнения, в то время как уравнение импульсов (7) представляет собой неинтегрируемое в замкнутом виде дифференциальное уравнение.

Введем функцию 


$$
\Phi=p F+\rho U^{2}\left(F-F_{1}^{*}-F_{2}^{* *}\right)
$$

и запишем уравнение импульсов в виде

$$
\frac{d \Phi}{d x}=p F^{\prime}(x)-\tau_{w} \chi .
$$

Из уравнения (4) выразим

$$
\rho=\frac{\dot{m}}{U F\left(1-\bar{F}_{1}^{*}\right)},
$$

тогда из (17) получим выражение для

$$
p=\frac{\Phi}{F}-U \frac{\dot{m}}{F} \frac{1-\bar{F}_{1}^{*}-\bar{F}_{2}^{* *}}{1-\bar{F}_{1}^{*}}
$$
для $U$ :

Подставляя отсюда $\rho$ и $р$ в уравнение энергии, получим следующее уравнение

$$
A U^{2}-B U+C=0
$$

где

$$
\begin{gathered}
A=\frac{1}{2}\left(\frac{k+1}{k-1}-\frac{2 k}{k-1}\left(\bar{F}_{1}^{*}-\bar{F}_{H}^{*}\right)\right) \frac{1-\bar{F}_{1}^{*}-\bar{F}_{2}^{* *}}{1-\bar{F}_{1}^{*}} \dot{m}, \\
B=\frac{k}{k-1} \Phi\left(1-\bar{F}_{1}^{*}+\bar{F}_{H}^{*}\right), \quad C=\dot{m} i_{0} .
\end{gathered}
$$

Физический смысл имеет корень уравнения (21) со знаком минус, то есть

$$
U=\frac{B-\sqrt{B^{2}-4 A C}}{2 A} .
$$

Таким образом, для определения параметров газа в ядре диффузора имеем систему уравнений

$$
\begin{gathered}
\frac{d \Phi}{d x}=p F^{\prime}-\tau_{w} \chi \\
\rho=\frac{\dot{m}}{U F\left(1-\bar{F}_{1}^{*}\right)}, \quad p=\frac{1}{F}\left(\Phi-\frac{U \dot{m}\left(1-\bar{F}_{1}^{*}-\bar{F}_{2}^{* *}\right)}{1-\bar{F}_{1}^{*}}\right) \\
U=\frac{B-\sqrt{B^{2}-4 A C}}{2 A}
\end{gathered}
$$


Напряжение трения на стенке $\tau_{w}$ определяется на основании методики, изложенной в [4]. Функции $\bar{F}_{1}^{*}, \bar{F}_{2}^{*}$ и $\bar{F}_{H}^{*}$ определяются из выражений (6), (9) и (11).

Обратим внимание на то, что при принятом подходе среди уравнений (23) лишь одно уравнение (для функции Ф) является дифференциальным, а остальные конечные.

Для интегрирования системы (23) совместно с соответствующими уравнениями для пограничного слоя нужны начальные условия в каком-либо сечении. Отметим, что для системы (23) и системы уравнений пограничного слоя безразлично в каком направлении интегрировать: вдоль скорости или против скорости.

Расчеты по потерям полного давления в диффузоре представлены на рис. 2. В качестве параметров при расчете были взяты коэффициент расхода $f$ (отношение действительного расхода воздуха к максимально возможному) и число Маха набегающего потока $M_{\infty}$. Параметры атмосферы взяты на уровне поверхности моря: $p_{\infty}=1,013 * 10^{5} \Pi a, \rho_{\infty}=1,226 \kappa 2 / \mu^{3}, T_{\infty}=288^{\circ} \mathrm{K}$.

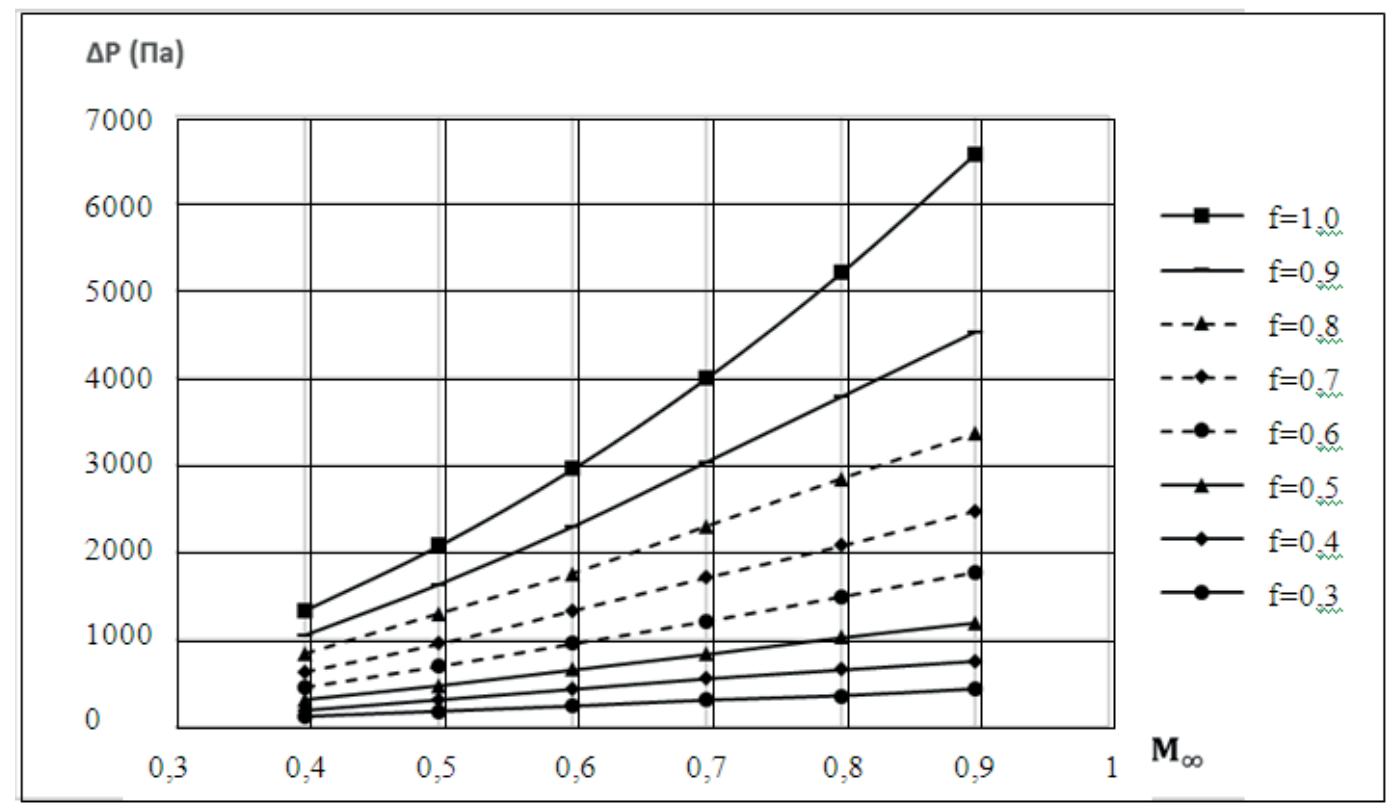

Рис. 2. Зависимость перепада давления между выходным и входным сечениями диффузора от числа Маха набегающего потока.

Приведенные результаты расчетов позволяют выбрать параметры диффузора, обеспечивающего необходимый расход и рассчитанного на определенную величину давления в выходном сечении.

\section{Библиографические ссылки}

1. Абрамович, Г. Н. Прикладная газовая динамика [Текст] / Г.Н. Абрамович. - М., Наука, 1976. -888 с. 
2. Шлихтинг, Г. Теория пограничного слоя [Текст] / Г. Шлихтинг. - М., Наука, 1969. $-742 \mathrm{c}$.

3. Дейч, М. Е. Техническая газодинамика [Текст] / М. Е. Дейч. - М., Энергия, 1974. $-592 \mathrm{c}$.

4. Вулис, Л. А. Термодинамика газовых потоков [Текст] / Л. А. Вулис - М., Гостехиздат, 1950. - 304 с.

5. Степанов, Г. Ю. Квазиодномерная газодинамика сопел ракетных двигателей [Текст] / Г. Ю. Степанов, Л. В. Гогиш - М., Машиностроение, 1973. - 168 с.

6. Гиневский, А. С. О расчете гидравлического сопротивления каналов [Текст] / А. С. Гиневский // ИФЖ, 1965, Т. 8, №4. - С. 540 - 545.

Надійшла до редколегії 20.08.2019 